\title{
Modified Citrus Pectin Supplement
}

\author{
National Cancer Institute
}

\section{Source}

National Cancer Institute. Modified Citrus Pectin Supplement. NCI Thesaurus. Code C103178.

A dietary supplement containing the modified citrus pectin (MCP) derived from the soluble fiber of citrus fruit peels and a galectin-3 inhibitor with potential antioxidant, hypocholesterolemic, immunostimulatory, metal chelating, and anti-metastatic activities. MCP is a low molecular weight version of pectin composed of short, slightly-branched carbohydrate chains and is modified for enhanced absorbability. The bioactive fragments, most likely the galactan-containing portion, of pectin binds to galectin-3, a carbohydrate-binding protein involved in imflammation, heart disease and is upregulated on the surface of certain types of tumor cells. Binding of MCP may result in the suppression of cancer cell aggregation, adhesion, proliferation and metastasis. In addition, MCP decreases prostate specific antigen (PSA) levels and may remove heavy metals. Also, unsaturated oligogalacturonic acids in MCP may stimulate the immune system through the activation of natural killer cells, cytotoxic T-cells, and B-cells. 